\title{
GSM OR X10 BASED SCADA SYSTEM FOR INDUSTRIAL AUTOMATION
}

\author{
Smita Suresh Wale, S.B. Patil \\ ME (ETX) Student, Dr.J.J.Magdum College of Engineering, Jaysingpur, Dist.-Kolhapur, State-Maharashtra \\ smitawale@yahoo.co.in
}

\begin{abstract}
Power systems are important and expensive components in the electric power system. The knowledge of the actual status of the system insulation behavior, load tap changer performance, temperature, and load condition is necessary in order to evaluate the service performance concerning reliability, availability and safety. Systems abnormalities, loading, switching and ambient condition normally contribute towards accelerated aging and sudden failure. The paper presents the causes which lead to the internal faults appearance in the power system. The production mechanisms of the faults and the on-line monitoring are also analyzed. A monitoring procedure is proposed for the diagnosis and forecasting strategy of the functioning state of the power system.
\end{abstract}

Keywords: GSM standard reference manual, Universal IC programmer

****

\section{INTRODUCTION}

A Supervisory Control and Data Acquisition system has been developed with an auto fault detection capability.[1] The Programmable Logic Controller used in a standard Supervisory Control and Data Acquisition system has been eliminated by using a microcontroller, resulting in a lower cost. Signals derived from a large number of detectors and sensors can be processed simultaneously.[2][3] The design enables the system to check the alarm status, true or false i.e. normal, faulty sensor, or open circuit. The data acquisition circuit is interfaced with the microcontroller board to evaluate the current flow conditions, normal or abnormal. A program written in Visual Basic provides for a graphical layout of the plan of the plant or building to be displayed on the monitor with a clear indication of the alarm spot.[1] A Close Circuit Television camera is made to move automatically to capture the particular location of the triggered alarm at the same time of the alarm signal. The methodological framework for managing Supervisory Control and Data Acquisition systems with auto fault detection using microcontroller constitutes the major contribution of the this.[2]

The power-line communications has been applied as a data transfer method in mechanical system and indoor distribution networks.[3] The characteristics of power-line channels and the applicability of different digital modulation techniques have been widely researched. Fault detection in mechanical system is an important element for industrial growth \& social development. For the last fifty years, electric power system has rapidly grown. This has resulted in large increase of the number of lines in operation \& their total length. These lines experience faults which are caused by insulation breakdown \& short circuits, sudden outage of line etc.[3][4]
The purpose of this project is to acquire to remote electrical parameters and send these real time values over Global System for Mobile Communication using Global System for Mobile Communication modem/phone.[1] The project is also design to protect the electrical circuitry by operating electromagnetic relay this relay gets activated whenever the electrical parameter exceed the predefined values, the relay can be used to operate the circuit breaker to switch off the main electrical supply.[2]

User can send commands in the form of SMS messages to read the remote electrical parameters. The system also can automatically send the real time electrical parameters periodically based on time setting in the form of SMS.[3] This also can be design to send SMS alerts whenever the circuit breakers trips or whenever the voltage and currents exceeds the predefined limits.[4]

\section{ARCHITECTURE}

Block Diagram

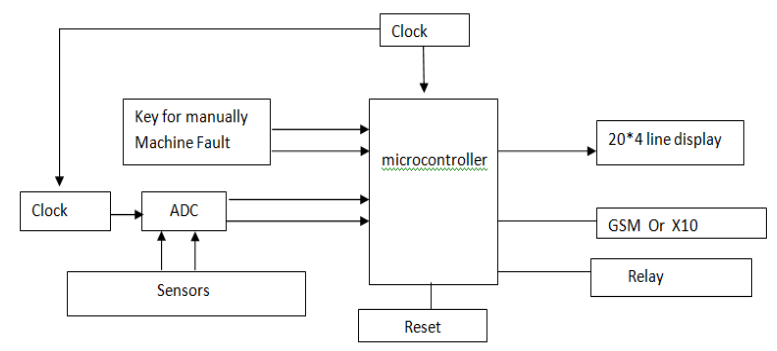

Fig 1 Transmitter 
1] MCU: (microcontroller unit)

It is the heart of system. All the controlling functions, data transmitting function are done by this unit. Following functions will be carried out by microcontroller unit.

1. Check whether any key is active or not if active then inform the fault related with the key to monitoring station via sms or $\mathrm{x} 10$ protocol.

2. To read ADC \& compare ADC data with set point \& if result is above set point then send the SMS to server also operate relay

2] GSM module:-

GSM SIM 300 is used as a media for communication between machine or user \& server. Server will be informed about fault via SMS. AT commands will be used.

3] X10 Module:-

It is used to send the data via a available power lines. It is plc for power line communication.

4] Sensors:

- Vibration sensor:-

Used to detect any vibration of machine

- CT sensor:-

To sense the current consumption

- Temperature sensor:-

To check the temperature of machine

5] Power supply requirement:

Power supply required is $12 \mathrm{v} \mathrm{dc} / 2 \mathrm{~A}$ max. For all system.

6] LCD:

$20 * 4$ line LCD is used to monitor the conditions of vehicle.

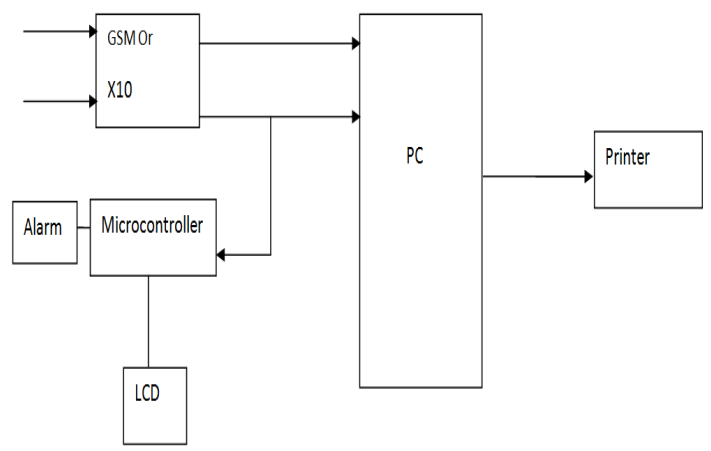

Fig 2 Receiver

Monitoring side:

1. At this side we will receive the information of different faults via SMS or x10 protocol.

2. This data is given to PC through gsm modem or $\mathrm{x} 10$ protocol via a max 232 converted to $\mathrm{PC}$

3. Pc will record the fault, fault time \& fault duration

$\&$ it will give the signal to trigger alarm to microcontroller. Record will be printed on user command.

This section describes the common features of the SCADA products that have been evaluated at CERN in view of their possible application to the control systems of the LHC detectors [1], [2].

\subsection{Hardware Architecture}

One distinguishes two basic layers in a SCADA system: the "client layer" which caters for the man machine interaction and the "data server layer" which handles most of the process data control activities. The data servers communicate with devices in the field through process controllers. Process controllers, e.g. PLCs, are connected to the data servers either directly or via networks or field buses that are proprietary (e.g. Siemens H1), or non-proprietary (e.g. Profile bus). Data servers are connected to each other and to client stations via an Ethernet LAN. The data servers and client stations are NT platforms but for many products the client stations may also be W95 machines. Fig.1 shows typical hardware architecture evolution as well as the potential benefits of their use.

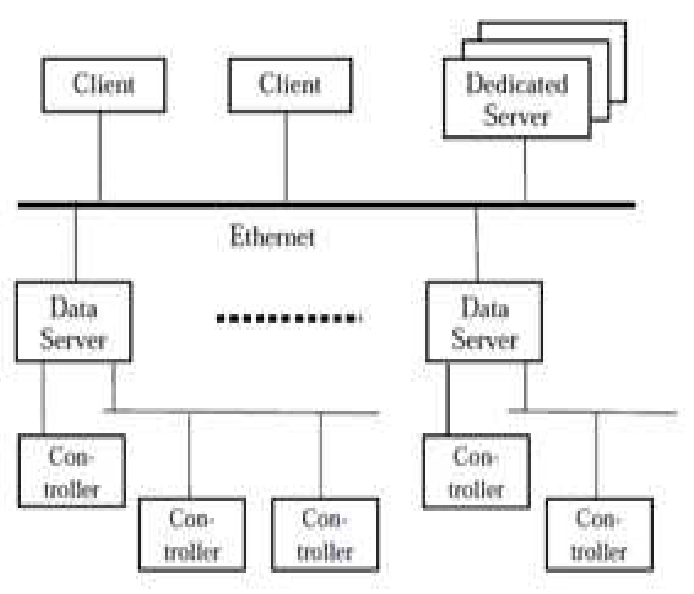

Fig 3.Typical hardware architecture

\subsection{Software Architecture}

The products are multi-tasking and are based upon a real-time database (RTDB) located in one or more servers. Servers are responsible for data acquisition and handling (e.g. polling controllers, alarm checking, calculations, logging and archiving) on a set of parameters, typically those they are connected to. 


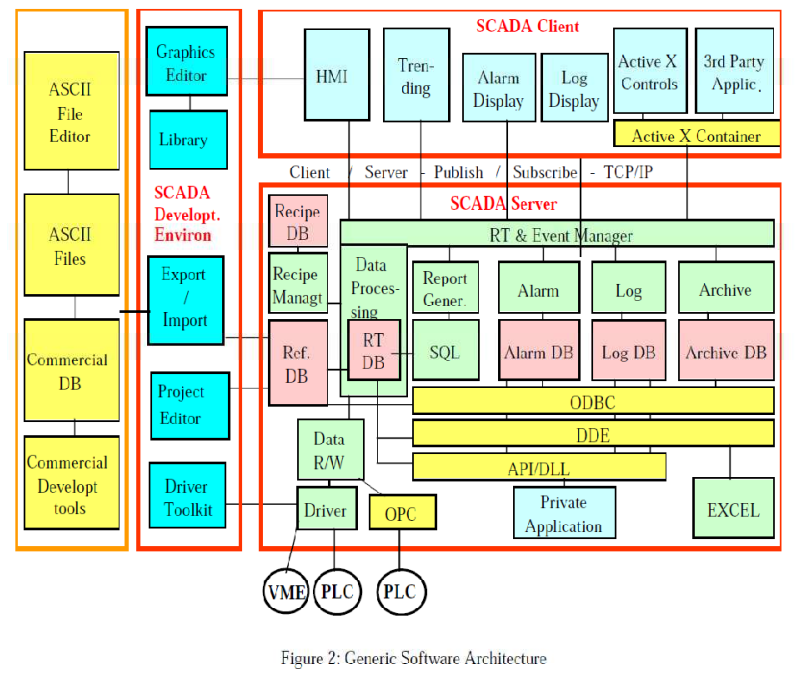

\section{GSM TECHNOLOGY}

One of the most important conclusions from the early tests of the new GSM technology was that the new standard should employ Time Division Multiple Access (TDMA) technology. This ensured the support of major corporate players like Nokia, Ericsson and Siemens, and the flexibility of having access to a broad range of suppliers and the potential to get product faster into the marketplace. After a series of tests, the GSM digital standard was proven to work in 1988.

With global coverage goals in mind, being compatible with GSM from day one is a prerequisite for any new system that would add functionality to GSM. As with other $2 \mathrm{G}$ systems, GSM handles voice efficiently, but the support for data and Internet applications is limited. A data connection is established in just the same way as for a regular voice call; the user dials in and a circuit-switched connection continues during the entire session. If the user disconnects and wants to re-connect, the dial-in sequence has to be repeated. This issue, coupled with the limitation that users are billed for the time that they are connected, creates a need for packet data for GSM.

The digital nature of GSM allows the transmission of data (both synchronous and asynchronous) to or from ISDN terminals, although the most basic service support by GSM is telephony.17 Speech, which is inherently analog, has to be digitized. The method employed by ISDN, and by current telephone systems for multiplexing voice lines over highspeed trunks and optical fiber lines, is Pulse Coded Modulation (PCM). From the start, planners of GSM wanted to ensure ISDN compatibility in services offered, although the attainment of the standard ISDN bit rate of $64 \mathrm{Kbit} / \mathrm{s}$ was difficult to achieve, thereby belying some of the limitations of a radio link. The $64 \mathrm{Kbit} / \mathrm{s}$ signal, although simple to implement, contains significant redundancy.
GSM is a typical $2 \mathrm{G}$ system in that it handles voice efficiently, but provides limited support for data and Internet applications. Operators frequently point to GSM penetration levels of more than $50 \%$ in order to justify required investments in $3 \mathrm{G}$ licenses, network construction, and services development. 8 That the extent of the costs of deployment for $3 \mathrm{G}$ has rendered it a 'costly business' is a tremendous understatement. This project makes use of an onboard computer which is commonly termed as microcontroller; this on board computer can efficiently communicate with the different sensors being used. The controller is provided with some internal memory to hold the code. This memory is used to dump some set of assembly instructions into the controller and the functioning of controller is dependent on these assembly instructions.

The project provides us exposure on

1) Initialization of ADC module of microcontroller

2) Embedded $C$ program

3) PCB designing

4) Different electrical sensors

5) Interfacing sensors to controller

6) LCD interfacing

The scope of this work is to research the applicability of power-line communications for industrial application data transfer required by the on-line condition monitoring system of mechanical system. Typically, energy consumed by consumer data transfer is performed between the controlling unit and simple devices, such as measuring devices or servers. The protocols used for data transfer are simple when they are compared to standard field bus protocols. The main objective of this work is: Measuring, the units consumed by power system through Power line communication.

The channel used for data transfer is low voltage distribution network. The topology of the network is dependent on the type and scale of the plant. Thus, the characteristics of the environment are also dependent on the type and scale of the plant.

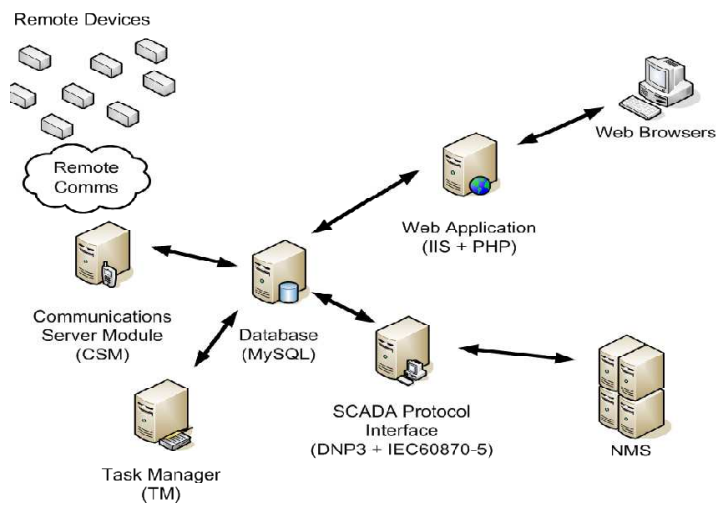

Fig 4 


\section{CONCLUSIONS}

This dissertation work covers practical applications and fault detection \& protection of power system \& keeps data record in data base to save time, money. To improve the performance and reduce the cost of a Supervisory Control and Data Acquisition system. To study and design a system that enables the status of the sensor to be detected. To improve the intelligence of the system by introducing auto fault diagnosis checking intervals can be preprogrammed to be every few seconds or minutes, to detect the change in sensor's state. To spot the particular alarm area by the camera and display the scene instantly on the computer, together with a floor plan indicating the exact location. This system makes use of microcontroller. It can efficiently communicate with the different sensors being used. The controller is provided with some internal memory to hold the code. This memory is used to dump some set of assembly instructions into the controller and the functioning of controller is dependent on these assembly instructions.

\section{RESULTS}

Faults like change in temperature, pressure and voltage will be detected by the sensors. The fault detection message will be sent to the control in charge's mobile phone or to control room. Also the current status of the parameter will be displayed on the LCD in real time.

\section{REFERENCES}

[1]. A Scada System Using Mobile Agents For A NextGeneration Distribution SystemTetsuo Otani, Member, IEEE, And Hiromu Kobayashi, Member, Ieee Transactions On Power Delivery, Vol. 28, No. 1, January 2013

[2]. Sensor Network Based Oilwell Health Monitoring and Intelligent Control Donglin Wang, Member, IEEE, Renlun He, Jiangqiu Han, Michel Fattouche, and Fadhel M. Ghannouchi, Fellow, IEEE sensors journal, vol. 12, no. 5, may 2012

[3]. Current Sensor Fault Detection, Isolation, And Reconfiguration For Doubly Fed Induction Generators Kai Rothenhagen, Member, IEEE, And Friedrich Wilhelm Fuchs, Senior Member, IEEE Transactions On Industrial Electronics, Vol. 56, No. 10, October 2009

[4]. Microcontroller Based Fault Detection Using Redundant Sensors Hector P. Polenta, Asok Ray, Sensor Member, And John A. Bernard, Sensor Member, IEEE Transactions On Industry Applications, Vol. 24. No.5.September/October 1988

\section{BIOGRAPHIES}

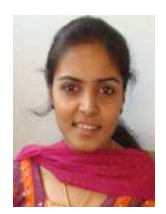

Miss. Smita Suresh wale, ME (ETX) Student, Dr.J.J.Magdum College of Engineering, Jaysingpur, Dist.-Kolhapur, State- Maharashtra smitawale@yahoo.co.in 